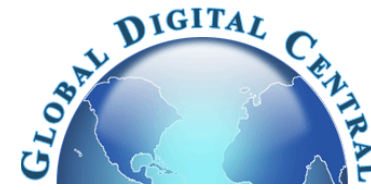

Frontiers in Heat Pipes

Available at www.HeatPipeCentral.org

\title{
FLAT PLATE HEAT PIPES: FROM OBSERVATIONS TO THE MODELING OF THE CAPILLARY STRUCTURE
}

\author{
Frédéric Lefèvre $^{\mathrm{a}, \mathrm{b}, \mathrm{c}, *}$, Stéphane Lips ${ }^{\mathrm{a}, \mathrm{b}, \mathrm{c}}$, Romuald Rullière ${ }^{\mathrm{a}, \mathrm{b}, \mathrm{c}}$, Jean-Baptiste Conrardy ${ }^{\mathrm{a}, \mathrm{b}, \mathrm{c}}$, Martin Raynaud ${ }^{\mathrm{d}}$, Jocelyn \\ Bonjour ${ }^{\mathrm{a}, \mathrm{b}, \mathrm{c}}$ \\ ${ }^{a}$ Université de Lyon, CNRS \\ ${ }^{b}$ INSA-Lyon, CETHIL, UMR5008, F-69621, Villeurbanne, France \\ ${ }^{c}$ Université Lyon 1, F-69622, France \\ ${ }^{d}$ Thales Communications, Thales, 92701 Colombes, France
}

\begin{abstract}
This paper presents a synthesis of observations made inside six different flat plate heat pipes (FPHPs). Silicon and copper systems with different capillary structures and sizes are studied. They are hermetically sealed on their upper face with a transparent plate, which enables liquid-vapor interface observations. A confocal microscope is used to measure the liquid film shapes inside the systems and describe the meniscus curvature radius from the evaporator to the condenser. Physical mechanisms, including the boiling limit and the effect of the interfacial shear stress, involved in different capillary structures such as grooves or meshes are discussed. Experimental results are compared to an analytical model describing both the liquid and vapour flows inside the FPHP and coupled to an analytical solution for the wall temperature. The capillary structure is modeled by considering the permeability and the equivalent thermal conductivity of a porous medium. The comparison between the model and the experimental data makes possible the estimation of the physical properties of the capillary structure. These experimental parameters are introduced in the model to estimate the performance of the heat pipe in a real application with several electronic components.
\end{abstract}

Keywords: confocal microscopy, grooves, meshes, electronic cooling, meniscus

\section{INTRODUCTION}

The interest in the use of heat pipes for thermal management of electronic components is recognized for both aerospace and terrestrial applications, especially with increasing heat flux requirements. In many applications, heat pipes are circular and are used to transport heat from one single heat source to one single heat sink. Flat plate heat pipes (FPHP) have the same function, but offer a wide cross-section, which enables the reduction of their thickness without reducing their thermal performance. They are particularly interesting when several heat sources have to be cooled, such as several electronic components on an electronic card. The thermal performance of a FPHP depends mainly on the capillary structure, which is usually made of grooves, meshes or sintered powder. FPHP can also be realized using several micro heat pipes placed parallel one to each other at a small distance.

Flat plate heat pipes (FPHP) have been widely studied in the last fifteen years, especially for their applications to electronic cooling (Lallemand and Lefèvre, 2004; Vasiliev et al., 2008). Although the literature on FPHP is abundant, experimental papers are relatively rare and most of the time do not provide measurements inside these systems, but only surface temperature measurements. Besides, theoretical works are generally focused on capillary-driven flows and thermal models in ideal conditions, i.e. the system being optimally filled with liquid (Khrustalev and Faghri, 1995; Kim et al., 2003; Lefèvre et al., 2008).

Since a few years, we developed experimental benches to visualize the flow in the capillary structure of FPHPs closed with transparent plates. Different capillary structures were studied (grooves with constant or variable width, crossed grooves, grooves covered with screen meshes, meshes) for FPHPs made of copper or silicon. Depending on the applications, we tested FPHPs with different sizes and different evaporator, condenser and adiabatic areas. The behavior of those systems filled with various working fluids were studied for different filling ratios and a wide range of applied heat fluxes.

Confocal microscopy has been used with varying degrees of success (depending on various parameters such as the size of the capillary structure, the heat flux applied at the evaporator, ...) to characterize the liquid-vapor interface in the capillary structure (Rullière et al., 2007; Lips et al., 2010a,b,c and 2011) and thin films of condensation on the groove fins (Lefèvre et al., 2010). Such measurements had never been provided before although they are necessary to validate most of the theoretical works published on FPHP (Lefèvre et al., 2008; Revellin et al., 2009). Temperature measurements are indeed not sufficient to validate hydrodynamic models that depend mostly on the meniscus curvature radius variation from the condenser to the evaporator. Furthermore, the temperature field in flat heat pipes depends on the thickness of thin films in both the condensing and evaporating areas (Lips et al., 2010a). Thus, the curvature radius of the menisci is a key parameter in both hydrodynamic and thermal models of heat pipes.

While confocal microscopy has been very useful to characterize FPHPs, visual observations have been sufficient to describe different physical phenomena of high importance to understand how FPHPs work. In the first sections of this paper, a synthesis of our observations is presented as well as what they have brought to the knowledge on these systems.

\footnotetext{
${ }^{*}$ Corresponding author.Email: Frederic.lefevre@insa-lyon.fr
} 
Physical phenomena involved in FPHPs are complex: evaporation and condensation phenomena, in presence of thin liquid films, in capillary geometries, and possibly boiling heat transfer. Models including such a complex physic are useful for the understanding but not convenient for engineering applications. In this paper, we also present results obtained with a very simple model (Lefèvre and Lallemand, 2006), in which the FPHP is characterized by its geometrical parameters, the properties of the capillary structure (permeability, equivalent thermal conductivity and effective radius), and the thermophysical properties of the wall and the working fluid. This model is particularly convenient for engineers since it enables the calculation of both the 3D temperature field and the capillary limit of a FPHP used to cool several electronic components (or any kind of heat sources) and several heat sinks, whatever their locations and their heat transfer rate.

In the last section of the article, the model is compared to experiments realized with a FPHP in order to estimate the permeability and the equivalent thermal conductivity of its capillary structure. The estimated properties are compared to analytical classical laws of the literature. Finally, these experimental parameters are introduced in the model to simulate the cooling of a real electronic card with several electronic components.

\section{EXPERIMENTAL BENCHES DEVELOPED TO STUDY FPHPs}

This section presents the physical and geometrical characteristics of different FPHPs and the associated experimental benches that were developed to characterize these systems.

\subsection{Six different flat plate heat pipes}

Six flat plate heat pipes, with different sizes and capillary structures have been tested. They are hermetically sealed on their upper face with a transparent plate, which enables liquid-vapor interface observations. Five of them are made of copper and one of silicon. In all cases, temperature sensors are located in the wall to measure the temperature field in working conditions.

FPHP 1, shown in Fig. 1, is made of a copper plate of thickness 1.5 $\mathrm{mm}$ and surface $267 \times 115 \mathrm{~mm}^{2}$ (Lefèvre et al., 2011 and 2012). The capillary structure, of dimensions $230 \times 90 \mathrm{~mm}^{2}$, is made of one or two CuSn 325 square screen mesh layers. The capillary screen meshes are plated against the copper plate by means of a coarse screen mesh, which creates the vapor space (Fig. 2). The heat source is a thick resistor film of dimensions $55.1 \times 96.5 \mathrm{~mm}^{2}$ and resistance $90.6 \Omega$. Two different heat sinks were tested: either two aluminum radiators $\left(140 \times 170 \mathrm{~mm}^{2}\right)$, cooled by natural convection of air at ambient temperature were screwed to each extremity of the heat pipe by thermal wedge locks or water heat exchangers of heat transfer area $30 \times 90 \mathrm{~mm}^{2}$ were plated against the wall also at each extremity.

FPHP 2 (Fig 3) is made of 109 longitudinal micro-grooves, machined in a copper plate of dimensions $230 \times 90 \mathrm{~mm}^{2}$ (Rullière et al., 2007; Lefèvre et al., 2008). Unlike classical FPHP applications, in which the heat source is small compared to the adiabatic and the heat sink regions, this FPHP has been tested with a large heat source $\left(190 \times 90 \mathrm{~mm}^{2}\right)$, a small heat sink $\left(30 \times 90 \mathrm{~mm}^{2}\right)$ and a small adiabatic region $\left(10 \times 90 \mathrm{~mm}^{2}\right)$. The application was the cooling of fuel cells. Each groove has a square cross-section of height and width equal to $400 \mu \mathrm{m}$. The distance between two grooves is equal to $400 \mu \mathrm{m}$. Good thermal performance was obtained with FPHP 2 in horizontal and in vertical favorable positions, but it was not able to work in unfavorable tilted position because capillary pressure induced by the grooved capillary structure was too low to overcome the hydrostatic pressure drop in vertical position. In order to overcome this limit, screen meshes were plated against the grooves (Lefèvre et al., 2012) to increase the capillary pressure gradient while preserving the high permeability of the grooves. FPHP 3 is similar to FPHP 2, but the grooves are covered by two layers of meshes (Fig. 4).
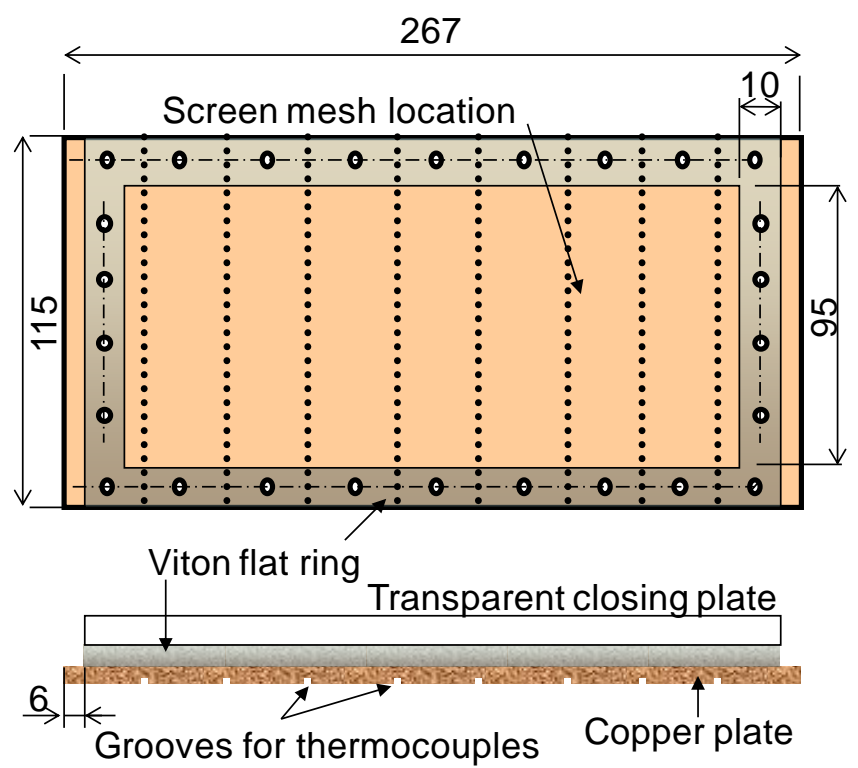

Fig. 1 Schematic of FPHP 1 (Lefèvre et al., 2012); length in mm
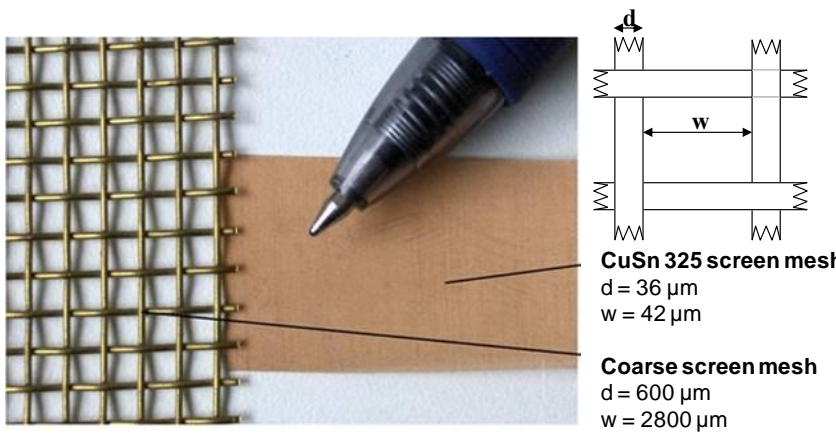

Coarse screen mesh $\mathrm{d}=600 \mu \mathrm{m}$ $w=2800 \mu m$

Fig. 2 Coarse and fine screen meshes (Lefèvre et al., 2012)

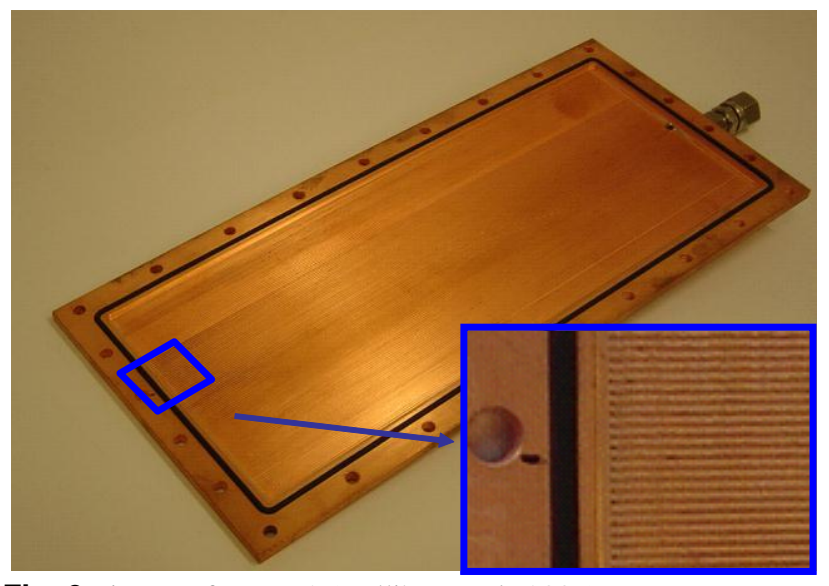

Fig. 3 Picture of FPHP 2 (Rullière et al., 2007)

FPHP 4 is also made of copper with the same grooves as FPHP 2 (Lips et al., 2009 and 2010). Compared to the previous systems, the dimensions are close to those encountered in electronic cooling applications (overall dimensions of $70 \times 90 \mathrm{~mm}^{2}$, evaporator and condenser area of $20 \times 90 \mathrm{~mm}^{2}$ and $30 \times 90 \mathrm{~mm}^{2}$ respectively).

The fifth heat pipe, FPHP 5, of dimensions $72 \times 68 \mathrm{~mm}^{2}$, is shown in Fig. 5. It is made of $85 \times 90$ crossed micro-grooves (that can also be viewed as an array of posts) machined in a copper plate. As for FPHP 2 , 3 and 4 , each groove has a square cross section $\left(400 \times 400 \mu \mathrm{m}^{2}\right)$ and the distance between two grooves is equal to $400 \mu \mathrm{m}$.

The sixth and last heat pipe, FPHP 6 (Fig. 6) is made of silicon. Its capillary structure, of diameter $50 \mathrm{~mm}$, is etched on a silicon wafer of 
$100 \mathrm{~mm}$ diameter and $525 \mu \mathrm{m}$ thickness. It is made of 72 radial microgrooves that are connected by crossed grooves at the centre of the device. The etching depth is equal to $200 \mu \mathrm{m}$. The radial grooves have a rectangular cross-section whose width varies from $670 \mu \mathrm{m}$ at the periphery to $110 \mu \mathrm{m}$ at the connection with the crossed grooves. These crossed grooves have a width equal to $100 \mu \mathrm{m}$ and they are separated by fins of same width, which creates a network of 300 small posts of $100 \times 100 \mu \mathrm{m}^{2}$

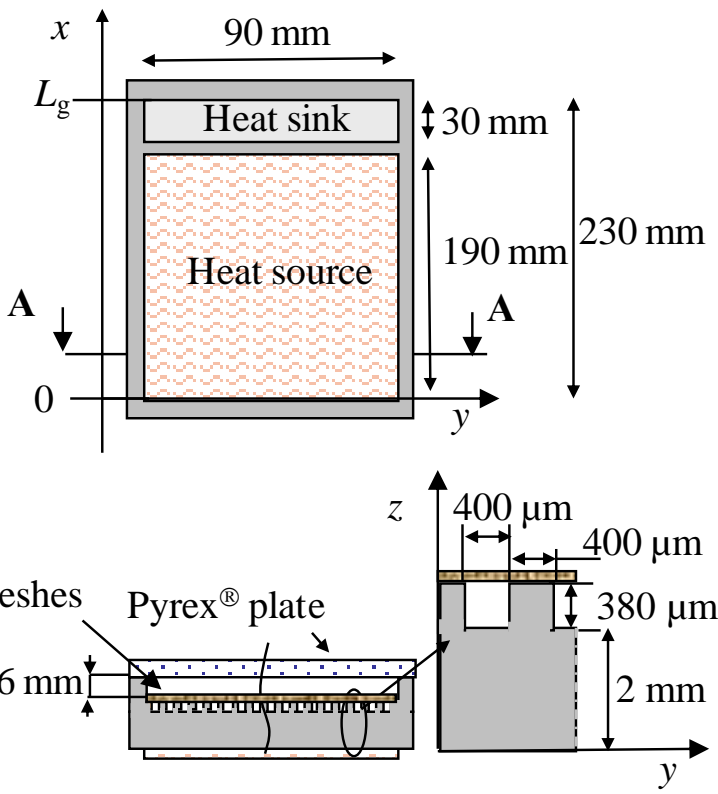

Cross-section A-A

Fig. 4 Schematic of FPHP 2 (grooves) or FPHP 3 (grooves and meshes)

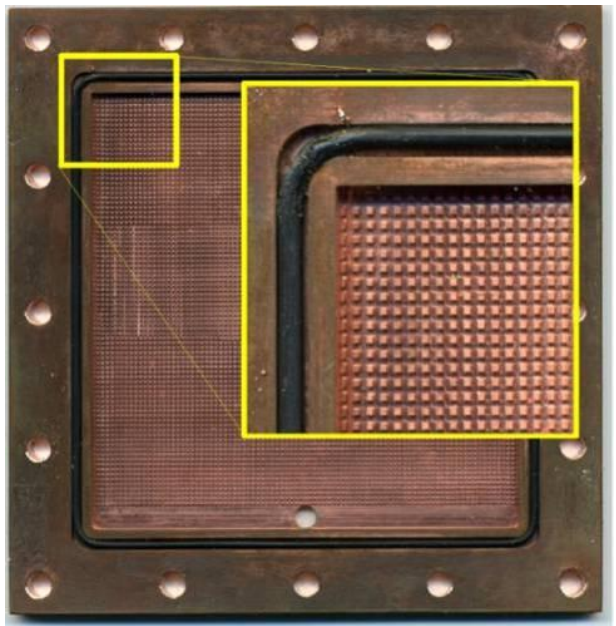

Fig. 5 Picture of FPHP 5 (Lips et al., 2010c)

\subsection{Confocal Microscopy}

Capillary phenomena, evaporation, and condensation in micro channels have been the focus of many experimental papers these last years. In a heat pipe, these phenomena are linked together and cannot be studied separately. Therefore, it is difficult to perform measurements in such a device. For example, micro-PIV is not helpful in heat pipes because the process of insemination is not convenient. Indeed, any particles introduced in the liquid would be blocked at the evaporator because of their non evaporative nature. Among classical techniques for film thickness measurement applied to micro-scale two-phase flow systems (Tibiriça et al., 2009), confocal microscopy appears to be the most appropriate to characterize FPHPs.
The confocal microscope is a STIL Micromesure 2 system. The optical sensor has a nominal measuring range of $350 \mu \mathrm{m}$. The working distance is about $13 \mathrm{~mm}$. The maximum measuring angle for specular reflection is about $27^{\circ}$. The optical sensor velocity is about $1 \mathrm{~mm} \cdot \mathrm{s}^{-1}$, which is fast enough for not thermally disturbing the measurement. Once the top of the groove is detected by the sensor, the liquid-vapor interface is recorded. The resulting data are used to estimate the curvature of the surface.

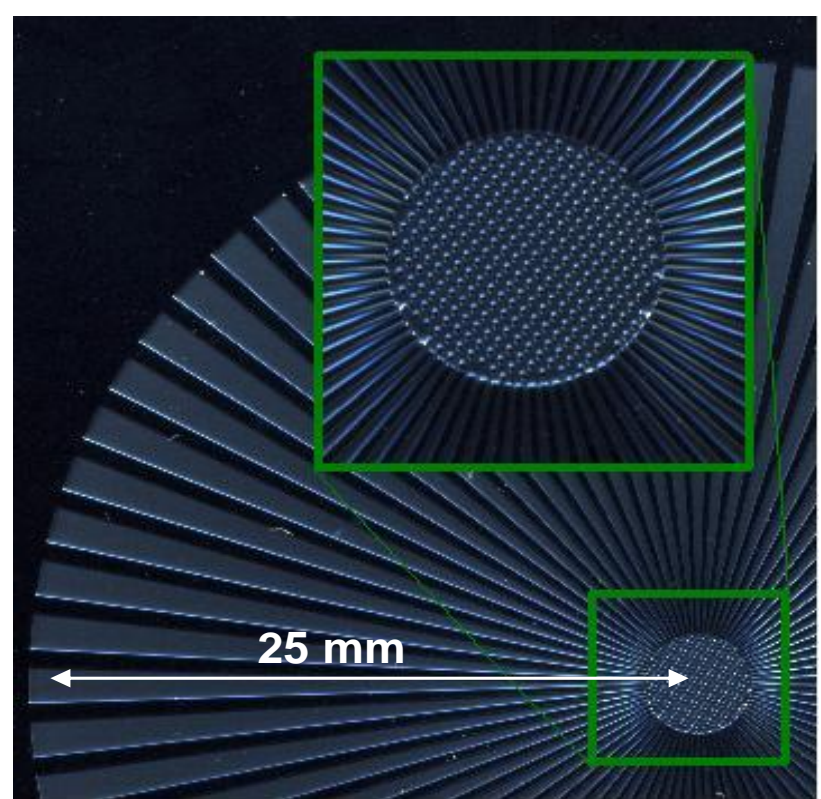

Fig. 6 Picture of FPHP 6 (Lefèvre et al., 2010)

Optical principles of confocal microscopy are described in Fig. 7. A white light point source is imaged by a lens that has not been corrected for chromatic aberration on a series of monochromatic point images in the measurement space. The reflected light passes back through the lens and is directed towards a spectral detector by a partially reflecting mirror. The spectral analysis of the collected lights that are separated by a spatial filter enables the location of the surface to be measured. The monochromatic light having the highest intensity corresponds to the surface to be measured.

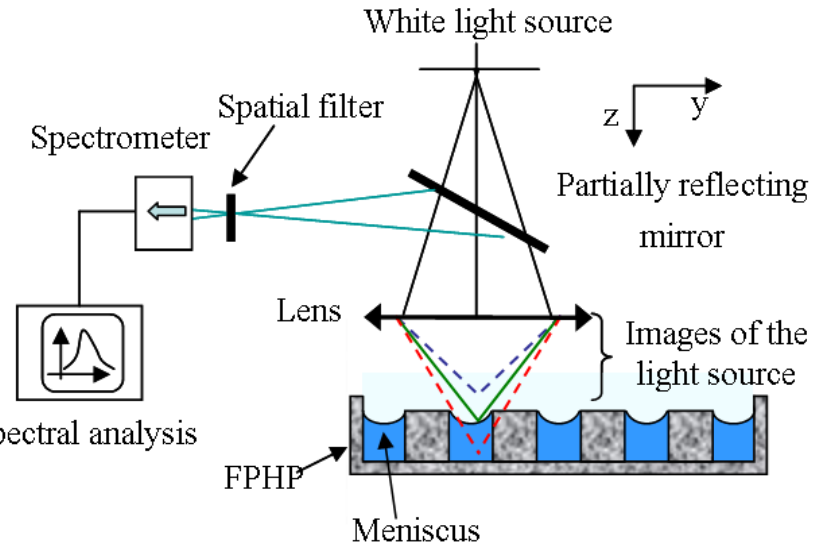

Fig. 7 Schematic diagram of confocal microscopy

\section{SYNTHESIS OF THE OBSERVATIONS INSIDE THE CAPILLARY STRUCTURE OF THE FPHPS}

\subsection{Examples of measurements}

Fig. 8 presents an example of measurement obtained with FPHP 6 , filled with methanol, in the condenser area. Two grooves are visualized. The width of the grooves and the fins are about $400 \mu \mathrm{m}$ and $800 \mu \mathrm{m}$, 
respectively (the scale on the $y$ axis is 100 times lower than that on the $x$ axis). This example shows that confocal microscopy permits to measure both the meniscus curvature radius inside the grooves and the condensate film on the fins. This last measurement is not possible with FPHPs made of copper. Indeed, the reflection of white light on a copper surface is larger than on silicon surface. As a result, the intensity of the reflected light is so high compared to that of the light reflected on the liquid-vapor interface that the focalization on the liquid film is not accurate enough for proper measurements. With silicon, the intensity of the reflected light is lower, which enables this measurement.

In example of Fig. 8, the thin condensate film thickness is lower than $5 \mu \mathrm{m}$. Circles that fit the experimental data are plotted. The radius of curvature is found to be $1.08 \mathrm{~mm}$ in the grooves and $12.4 \mathrm{~mm}$ on the fins. The curvatures between these zones are reversed. Therefore, the Young-Laplace law yields to the conclusion that the liquid pressure is higher than the vapor pressure on the fins and lower than the vapor pressure in the grooves. It has to be noted that, from our measurements, the transition between the two films looks abrupt. Thus, a very small region shall be present where the curvature changes from a constant positive value to a constant negative value. Such measurements are useful to understand evaporation and condensation processes that highly depend on the shape of the liquid-vapour interface (Lips et al., 2010a).

For FPHP 6, the microscope was not useful to measure the menisci in the part of the capillary structure where groove widths are to small $(<150 \mu \mathrm{m})$. Indeed, for such small grooves, the slope of the meniscus at the junction with the grooves is higher than the maximum measuring angle of the optical sensor. This is a real limitation of this measurement method.

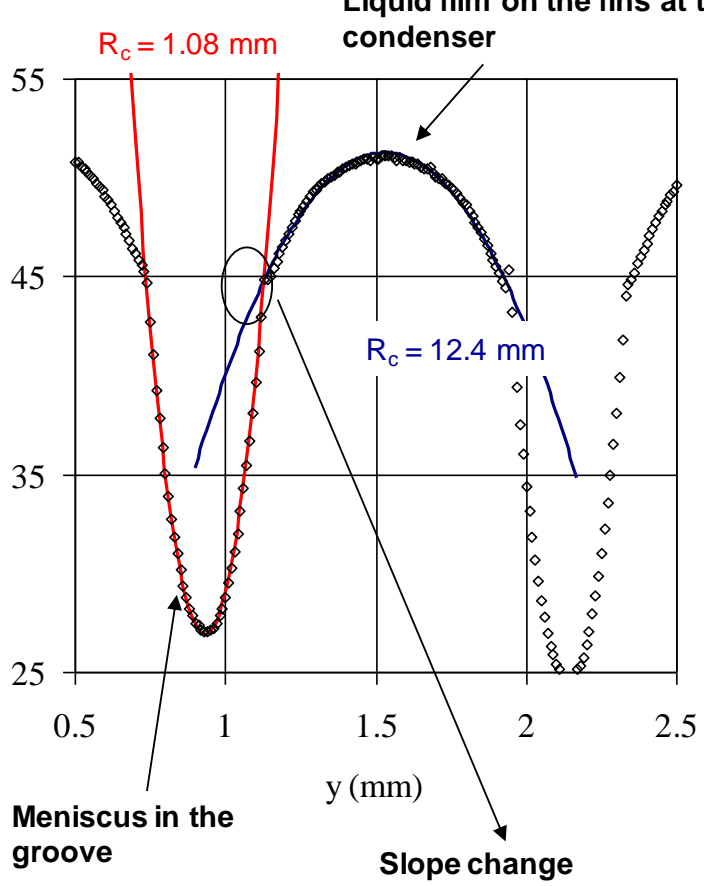

Fig. 8 Liquid-vapor interface FPHP 6 (Lefèvre et al., 2010)

Confocal microscopy has also been used to measure the liquid-vapor interface in crossed-grooves (FPHP 5). Fig. 9 presents a measurement obtained at the junction between two grooves. The top of the fins is not homogeneous because of the copper roughness. The liquid-vapor interface is smoother and has a saddle shape. As the measurement of the confocal microscope is punctual, the surface is obtained by moving the FPHP with a motorized table along $x$ and $y$ directions.

The surface curvature is identified from the experimental data. The capillary pressure $P_{c a p}$ is deduced from the Young-Laplace law. The measurement method can be validated in non working conditions, with the FPHP being tilted. In such conditions, the capillary pressure is theoretically equal to the hydrostatic pressure of the liquid. Fig. 10 presents the capillary pressure measured for FPHP 5 filled with methanol and with FC72, with an inclination angle with respect to the horizontal equal to $5^{\circ}$ and $2.5^{\circ}$ respectively. Solid lines are the hydrostatic pressures. A good agreement is observed between experimental and theoretical results. The accuracy of the method is evaluated to be of the order of $1 \mathrm{~Pa}$.

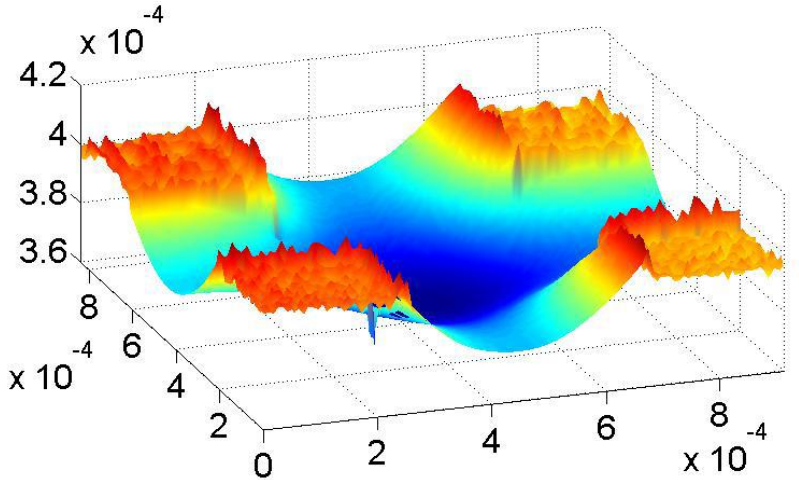

Fig. 9 Liquid-vapor interface in FPHP 5 (dimensions in m) (Lips et al., 2010c)

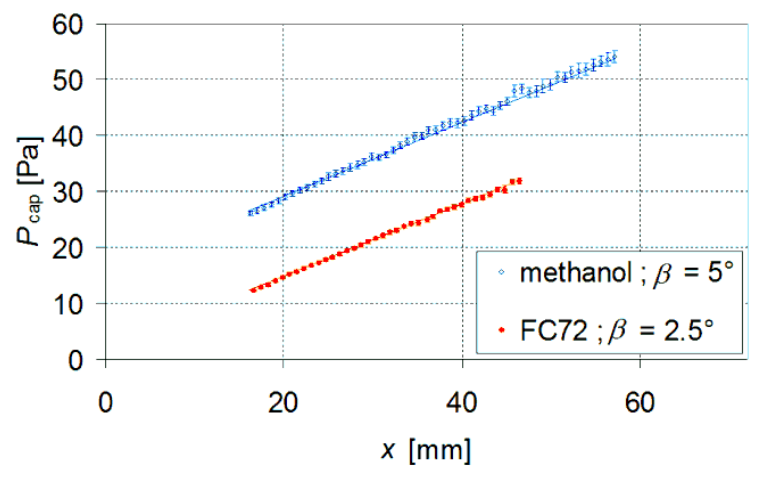

Fig. 10 Validation of the experimental method (Lips et al., 2010c)

\subsection{Synthesis of the information brought by the observations}

FPHP 2 and FPHP 4 have been studied in different experimental conditions (Rullière et al., 2007; Lips et al., 2010b) in order to obtain a significant experimental database. Experimental parameters are the vapor space thickness $(1 \mathrm{~mm}, 1.6 \mathrm{~mm}$ and $2 \mathrm{~mm})$, the inclination, the filling ratio, the heat flux transferred by the system (between $0.5 \mathrm{Wcm}^{-2}$ and $3 \mathrm{Wcm}^{-2}$ ) and the working fluid (N-pentane or methanol).

Fig. 11 presents the comparison between this database and a hydrodynamic model based on the balance equations and the YoungLaplace law (Lips et al., 2011). To facilitate and reduce the presentation of the hydrodynamic measurements, the experimental results obtained by confocal microscopy are presented in the form of the mean capillary pressure gradient $\Delta P_{c a p} / \Delta x$ in the adiabatic zone. The uncertainty of the capillary pressure gradient is given for each experiment. In all the cases, a good agreement is observed between experimental and numerical results. The numerical model tends to slightly overestimate the capillary pressure gradient in the system. The mean relative error of the model is about $15 \%$.

In theoretical models developed in the literature, shear stress calculation is often viewed as the most important parameter for pressure drop calculations. In the present model, the liquid-wall and vapor-wall shear stresses are calculated with a classical approach in laminar conditions (Faghri, 1995). In Fig. 11, interfacial shear-stress has been calculated considering the liquid as motionless for the vapor, but in all these experimental conditions, neglecting the interfacial shear stress does not change significantly the results (Lips et al., 2011), even with a 
small vapor space $(1 \mathrm{~mm})$. It has to be noted that these experimental conditions are close to those encountered in the literature (Lallemand and Lefèvre, 2004).

While interfacial shear stress has not a strong influence on FPHP performance, experimental results obtained by confocal microscopy with FPHP 4 show that FPHP performance is strongly influenced by both the filling ratio and the vapor space thickness (Lips et al., 2010). A small vapor space thickness induces liquid retention in the FPHP corners and sides and thus reduces the thermal resistance of the system. Nevertheless, the meniscus curvature radius at the condenser decreases with the decrease of the vapor space thickness, which reduces the maximum capillary pressure of the FPHP. As an example, a vapor space of $1 \mathrm{~mm}$ leads to a $35 \%$ decrease of the maximum capillary pressure with respect to a very large vapor space thickness.

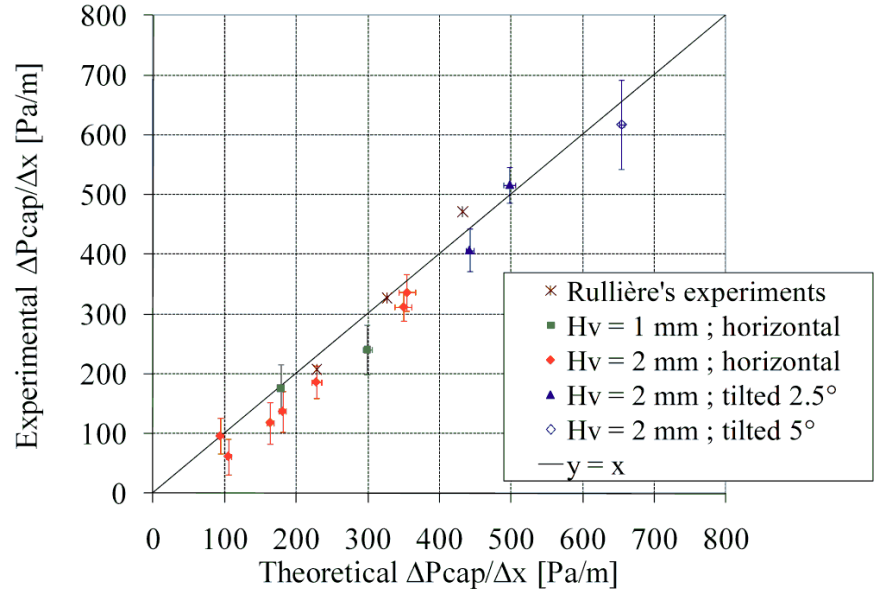

Fig. 11 Comparison between experimental and numerical hydrodynamic results (Lips et al., 2011)

Confocal microscopy is well adapted to characterize FPHP but visual observations are sufficient to describe different physical phenomena of high importance to understand how FPHP works. For example, nucleate boiling (Fig. 12) has been observed in various configurations for relatively small heat fluxes (Lips et al., 2009; Lefèvre et al., 2012).

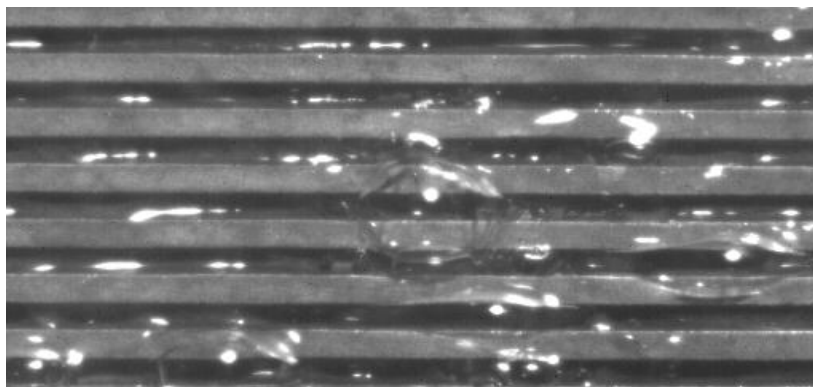

Fig. 12 Example of boiling phenomenon in the evaporator of FPHP 4 (Lips et al., 2009)

For FPHP 4, filled with methanol, nucleate boiling was observed for heat fluxes higher than $3 \mathrm{~W} / \mathrm{cm}^{2}$ and until $18 \mathrm{~W} / \mathrm{cm}^{2}$. Unexpectedly, this phenomenon does not prevent the working of the system and conversely increases its thermal performance. An experiment conducted in non horizontal orientation (Lips et al., 2011) showed that the presence of nucleate boiling in the system does not constitute a limit as it is generally stated in the literature. In all cases, dryout of the evaporator is due to the capillary limit. All these investigations have been made under normal gravity conditions. But heat pipes are often used in space applications: it would be interesting to perform identical tests under micro gravity, in order to determine if the conclusions of this study can be extended to this case. In any case, a better understanding of these phenomena is necessary, particularly to theoretically predict the operation limit in case of nucleate boiling. Nucleate boiling was also observed in FPHP 5 and 6 for similar heat fluxes. Conclusions were the same. For FPHP 2, this phenomenon was not observed because the evaporator area is very large and thus capillary limit was observed for heat fluxes lower than $1 \mathrm{Wcm}^{-2}$, before the onset of nucleate boiling.

On the contrary, the performance of the heat pipe obtained with the association of grooves and meshes (FPHP 3) is not as high as one could expect (Lefèvre et al., 2012) regarding the capillary limit. Indeed, while this capillary structure enables to work in tilted unfavourable position, which was not possible with the grooved heat pipe (FPHP 2), a clear nucleate boiling limitation is observed for rather small heat fluxes $\left(\sim 0.4 \mathrm{~W} / \mathrm{cm}^{2}\right)$. This dryout is brutal and takes place on all the surface of the FPHP, which was not the case for FPHP2 when the capillary limit was encountered. This dryout is probably due to the rapid expansion of bubbles, which grow between the screen mesh layer and the grooves, rather than outside of it, because of the capillary pressure barrier imposed by the meshes. As a result, bubble growth pushes away the liquid from the grooves. As the screen mesh layers are not able to drain the liquid on the entire surface, the capillary structure suddenly dries out after the boiling incipience.

The observations made inside FPHPs have increased considerably the knowledge on these systems. Confocal microscopy enables to measure the vapor and liquid pressure difference along the capillary structure. These measurements, associated with temperature measurements enable the estimation of the thermophysical properties of the capillary structure by comparison with an appropriate model.

\section{AN ANALYTICAL MODEL FOR THE COOLING OF MULTIPLE ELECTRONIC COMPONENTS}

This section presents a 2D analytical solution for both the liquid and vapor flows inside a FPHP coupled to a 3D analytical solution for the temperature inside the FPHP wall. The thermal model is able to calculate the part of the heat flux transferred only by heat conduction in the wall from the heat transferred by change of phase.

\subsection{Equations of the model}

The model was already described in previous articles (Lefèvre and Lallemand, 2006; Revellin et al., 2009) to which the reader should refer for further details.

The geometry of the FPHP is shown in Fig. 13. It consists of a flat plate of $a \times b$ rectangular dimensions. The electronic components and the heat sinks can be located anywhere on the FPHP and have different heat transfer rates. The rest of the surface is adiabatic. The wall of the FPHP is made of a material of thermal conductivity $\lambda_{s}$ and its height is equal to $c$. The capillary structure is modeled by considering a porous medium of permeability $K$, thermal conductivity $\lambda_{p}$ and height $H_{p}$. The liquid inside the porous medium has a dynamic viscosity $\mu_{l}$, a heat conductivity $\lambda_{l}$ and a density $\rho_{l}$. The equivalent thermal conductivity of both the liquid and the porous medium is equal to $\lambda_{e q}$. The vapor space height is equal to $H_{\mathrm{v}}$, and $\lambda_{v}$ and $\mu_{v}$ are the vapor thermal conductivity and dynamic viscosity, respectively. The thermal properties are supposed to be constant. They are calculated at the saturation temperature $T_{\text {sat }}$.

The 3D heat conduction equation is solved in steady state by considering adiabatic conditions in $x=0, x=a, y=0$ and $y=b$. In $z=c$, a heat flux, which depends on the location and the heat transfer rate of the heat sources and the heat sinks is imposed. In $z=0$, a Fourier boundary conditions is considered with a heat transfer coefficient function of both the thickness and the thermal conductivity of the capillary structure. By considering these boundary conditions, the temperature field $T(x, y, z)$ in the wall of the FPHP can be expressed by Fourier's series: 


$$
T(x, y, z)=C_{1} \sum_{m=0}^{\infty} \sum_{n=0}^{\infty} A_{m n} \cos \left(\frac{m \pi x}{a}\right) \cos \left(\frac{n \pi y}{b}\right)
$$

where $C_{l}$ is a constant and $A_{m n}$ are functions of the location and of the power of the heat sources and heat sinks. They also depend on the thickness and the equivalent thermal conductivity of the capillary structure.

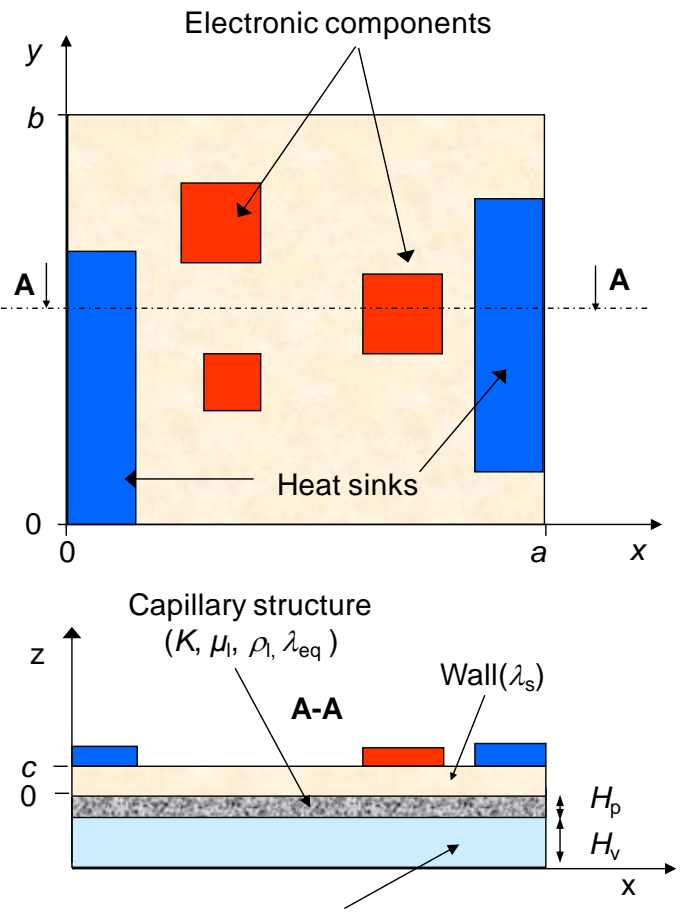

Vapor space $\left(\mu_{\mathrm{v}}, \rho_{\mathrm{v}}\right)$

Fig. 13 Schematic of the FPHP for the model (Lefèvre and Lallemand, 2006)

Once the temperature field is calculated, it is possible to calculate the evaporation and condensation mass flow rates at each point of the system by deriving the temperature field in $z=0$. A $2 \mathrm{D}$ hydrodynamic model, based on Darcy's law and on mass balance equations, is used to calculate the liquid and vapor velocities and pressures in the capillary structure. The velocities are equal to zero in $x=0, x=a, y=0$ and $y=b$. By considering these boundary conditions, the pressure field for both the liquid and the vapor, can also be expressed by Fourier's series:

$P(x, y)=C_{2} \sum_{m=0}^{\infty} \sum_{n=0}^{\infty} B_{m n} \cos \left(\frac{m \pi x}{a}\right) \cos \left(\frac{n \pi y}{b}\right)$

where $C_{2}$ is a constant depending on $\mu_{l}, \rho_{l}$ and $K$ for the liquid and $\mu_{v}$, $\rho_{v}$ and $H_{\mathrm{v}}$ for the vapor. $B_{m n}$ are functions derived from the thermal model.

The maximum heat transfer capability of the FPHP is obtained when the maximum capillary pressure is equal to the sum of the pressure drops in the liquid $\Delta P_{l}$ and the vapor $\Delta P_{v}$ and the hydrostatic pressure $\Delta P_{g}$ that can be either positive or negative, depending on the location of the heat sources and the heat sinks:

$\Delta P_{\text {cap }}=\Delta P_{l}+\Delta P_{v}+\Delta P_{g}$

The capillary pressure can be calculated using the following classical expression (Faghri, 1995), which results from the YoungLaplace law:

$\Delta P_{\text {cap }}=\frac{2 \sigma}{r_{\text {eff }}}$

where $r_{\text {eff }}$ is the effective pore radius and $\sigma$ is the surface tension of the fluid.

\subsection{Estimation of the permeability and the equivalent thermal conductivity by comparison with experimental data}

The comparison between the model and experimental data enables the estimation of both the permeability and the equivalent thermal conductivity of the capillary structure. When temperature measurements are available along the FPHP wall, the thermal conductivity is obtained when the model fits the experimental data. One can note that if the distance between the heat sources and the heat sinks is high enough, it is possible to differentiate the thermal conductivity at the condenser and at the evaporator (Revellin et al., 2009) using the same model. When pressure measurements (deduced from confocal microscopy measurements) are available, the permeability is obtained when the model fits the experimental data. Otherwise, an estimation of the permeability can be obtained by considering the heat transfer rate for which the dryout of the evaporator is reached. If the maximum capillary pressure of the capillary structure is known, an estimation of the permeability is obtained when the sum of the pressure drops in the liquid and the vapour, which is a function of the permeability, is equal to the capillary pressure.

As an example, we present in this section the comparison between the model and experimental results obtained with FPHP 1 (Lefèvre $e t$ $a l .$, 2012). The capillary structure is made of two CuSn 325 square screen mesh layers. The FPHP is filled with methanol. The heat source of surface $55.1 \times 96.5 \mathrm{~mm}^{2}$ is located in the middle of the FPHP. Two water heat exchangers of surface $30 \times 90 \mathrm{~mm}^{2}$ are located on each side of the system (Fig. 14).

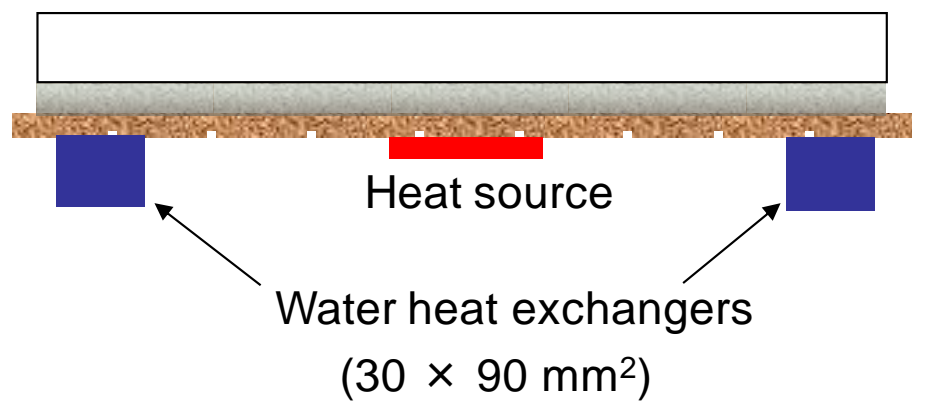

Fig. 14 Locations of the heat sink and the heat source on the FPHP 1

Fig. 15 presents the temperature profile in horizontal orientations for a heat transfer rate equal to $15 \mathrm{~W}$. Fig. 16 presents the maximal temperature difference between the heat source and the heat sinks in horizontal orientation vs. the heat transfer rate of the heat source. Linear characteristics are obtained until a threshold heat transfer rate of $82 \mathrm{~W}$. Below this value, the thermal resistance is equal to $0.14 \mathrm{~K} / \mathrm{W}$ and can be compared to the thermal resistance of the copper plate alone $(0.62 \mathrm{~K} / \mathrm{W})$.

In the model, the thickness of the capillary structure is set to 140 $\mu \mathrm{m}$, which corresponds to twice the thickness of a CuSn mesh screen layers. The maximum capillary pressure is calculated with Eq. (4) by considering that the effective radius is equal to half the distance $w$ between two fibers (Fig. 2). For methanol, $\Delta P_{c a p}$ is equal to about $2000 \mathrm{~Pa}$. In the model, the permeability is adjusted until the pressure drops in the liquid and the vapor, calculated for the threshold heat transfer rate of $82 \mathrm{~W}$, is equal to the capillary pressure. The permeability of the meshes is estimated to $0.55 \times 10^{-10} \mathrm{~m}^{2}$.

For a mesh with a fiber diameter equal to $d$ and a distance between two fibers equal to $w$ (Fig. 2), the permeability $K$ can be expressed as (Faghri, 1995): 


$$
K=\frac{d^{2} \varphi^{3}}{122(1-\varphi)^{2}}\left\{\begin{array}{l}
\varphi=1-\frac{1.05 \pi N d}{4} \\
N=\frac{1}{d+w}
\end{array}\right.
$$

where $\varphi$ is the porosity. For this mesh, $d=35 \mu \mathrm{m}$ and $w=42 \mu \mathrm{m}$. Thus, the permeability calculated with Eq. (5) is equal to $0.18 \times 10^{-10} \mathrm{~m}^{2}$, which is 3 times lower than that estimated experimentally.

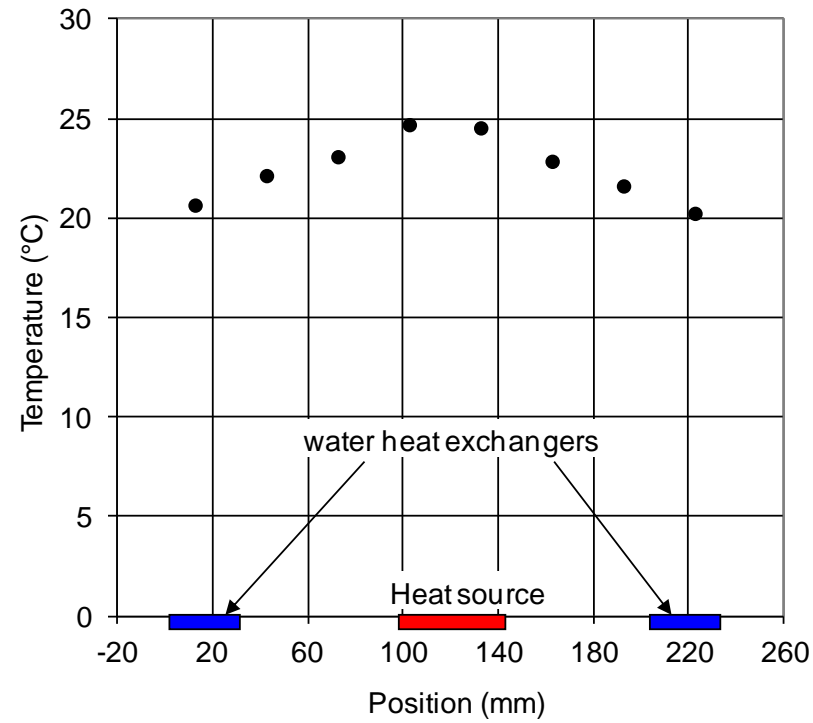

Fig. 15 Temperature field for a heat transfer rate of $15 \mathrm{~W}$ in horizontal location

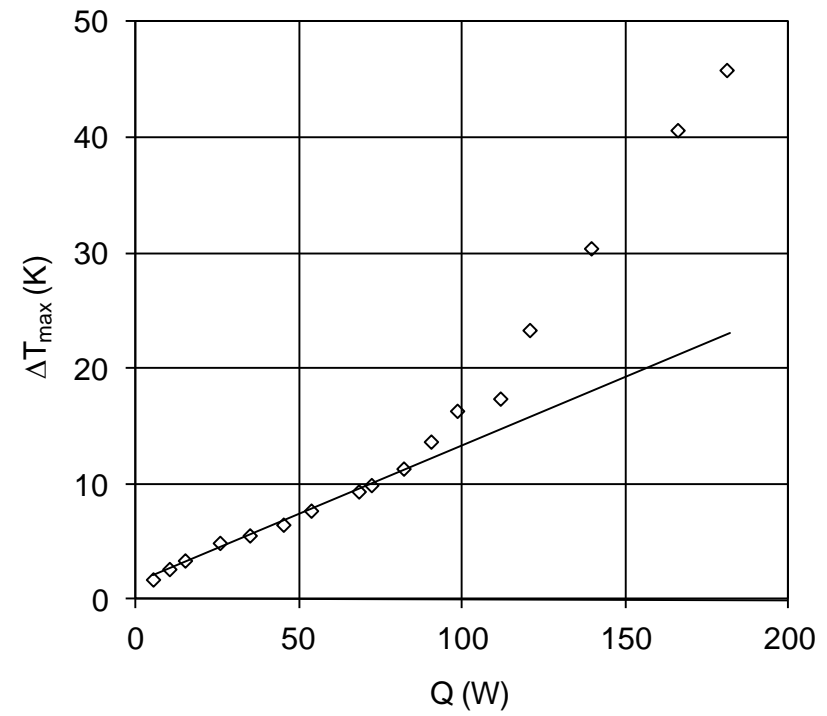

Fig. 16 Maximal temperature difference vs. heat transfer rate; horizontal location (Lefèvre et al., 2012)

Fig. 17 presents an example of the comparison between the model and the measured temperatures for a heat flux of $82 \mathrm{~W}$. The equivalent thermal conductivity that fits at best the experimental data is equal to $0.23 \mathrm{~W} / \mathrm{mK}$. It has to be noted that this equivalent thermal conductivity is close to that of methanol $(\lambda=0.2 \mathrm{~W} / \mathrm{mK})$.

For a mesh with a fiber diameter equal to $d$ and a distance between two fibers equal to $w$ (Fig. 2), the equivalent thermal conductivity depends on both the liquid and the mesh thermal conductivities $\lambda_{l}$ and $\lambda_{s}$ (Faghri, 1995):

$$
\lambda_{e q}=\frac{\lambda_{l}\left[\left(\lambda_{l}+\lambda_{s}\right)-(1-\varphi)\left(\lambda_{l}-\lambda_{s}\right)\right]}{\left[\left(\lambda_{l}+\lambda_{s}\right)+(1-\varphi)\left(\lambda_{l}-\lambda_{s}\right)\right]}
$$

The equivalent thermal conductivity calculated with Eq. (6) is equal to $0.44 \mathrm{~W} / \mathrm{mK}$, which is twice higher than that estimated experimentally.

The permeability estimated experimentally is 3 times higher than that calculated theoretically while the experimental equivalent thermal conductivity is 2 times lower. Both discrepancies can be partly explained by an underestimation of the real thickness of the screen meshes. Indeed, as the screen meshes are not sintered to the wall, the contact with the wall is most likely imperfect, which leads to an increase of the actual thickness of the capillary structure. However, the underestimation of the real thickness of the capillary structure is not sufficient to explain such important differences. The reliability of the usual laws for both the permeability and the equivalent thermal conductivity can be questioned, as it was already questioned for grooved capillary structures (Revellin et al., 2009). Lips et al (2010a) also showed experimentally that the usual laws strongly overestimate

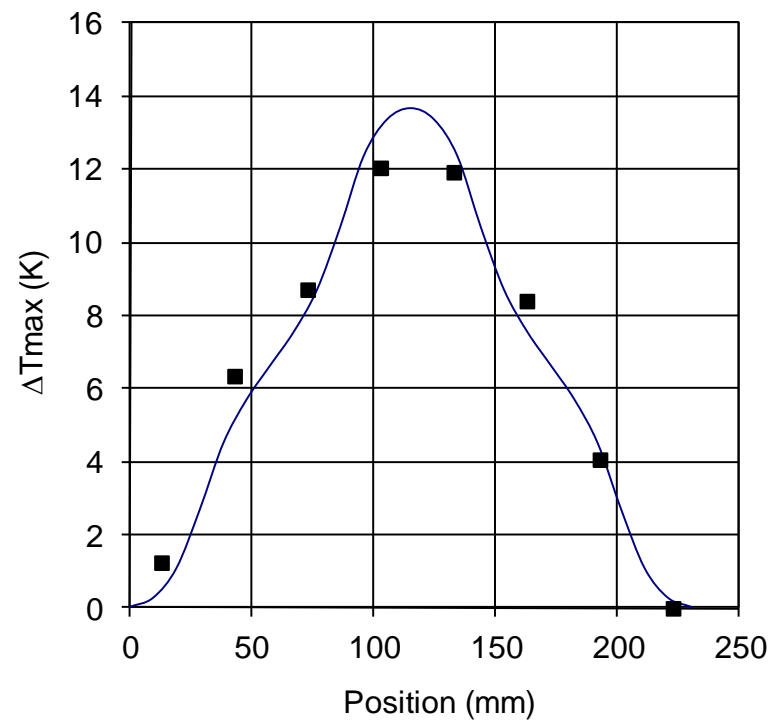

Fig. 17 Comparison between the analytical model and experimental data $\left(Q=82 \mathrm{~W} ; \lambda_{e q}=0.23 \mathrm{~W} / \mathrm{mK}\right)$

Finally, these different comparisons show that there is a real need for accurate equivalent thermal conductivity correlations for the different capillary structures encountered in practice. It has to be noted that for crossed grooves, Lips et al (2010c) could not compare their experimental thermal conductivity to such a theoretical law because no correlation is available in the literature for this kind of geometry. On the other hand, Lips et al (2010c and 2011) showed that satisfactory models are available for the determination of the permeability for grooved capillary structures. However, the present study emphasizes that it is not the case for capillary structure made of metallic meshes.

Fig. 18 presents the equivalent thermal conductivity of the capillary structure as a function of the heat transfer rate $Q$. These results have been calculated by comparing the analytical model to the experimental data of Fig.16. The equivalent thermal conductivity is rather constant for heat transfer rates between $45 \mathrm{~W}$ and $82 \mathrm{~W}$. It decreases beyond $82 \mathrm{~W}$, due to the beginning of the dryout of the capillary structure. For increasing heat fluxes from $Q=0 \mathrm{~W}$ to $Q=45$ $\mathrm{W}$, the thermal conductivity increases from a very small value $(0.07$ $\mathrm{W} / \mathrm{mK}$ ) to a constant value, which can be explained by a slight overfilling for small heat fluxes. 


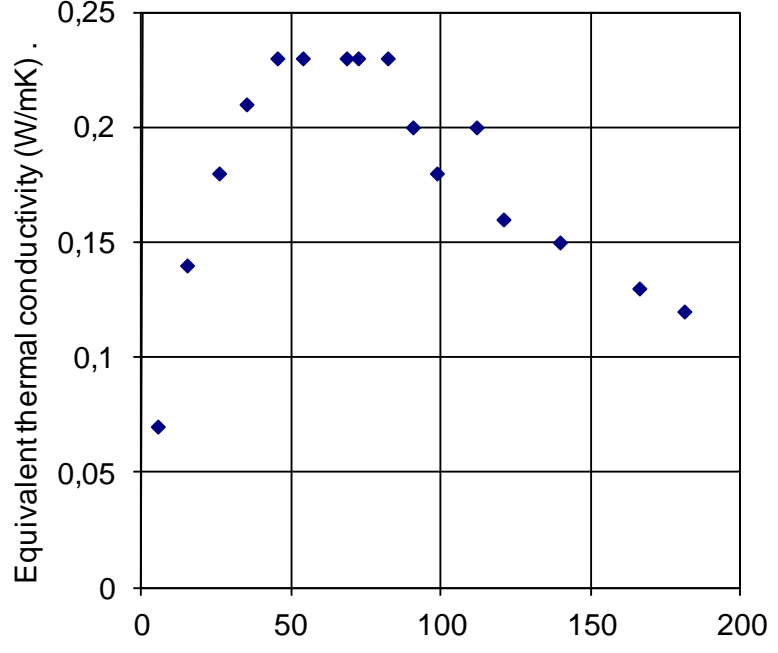

Heat transfer rate $(\mathrm{W})$

Fig. 18 Equivalent thermal conductivity versus power (estimated from experimental results of Fig. 16)

\subsection{Application to the cooling of several electronic components}

Fig. 19 presents the temperature field for the FPHP in horizontal orientation with three heat sources and two heat sinks located on its shorter edge. The properties of the capillary structure are those estimated in the previous sections, i.e. an equivalent thermal conductivity equal to $0.23 \mathrm{Wm}^{-1} \mathrm{~K}^{-1}$ and a permeability of $0.55 \times 10^{-10}$ $\mathrm{m}^{2}$. The heat sink size and location are the same as the water heat exchangers on each side of the FPHP. The total heat transfer rate is equal to $75 \mathrm{~W}$ and is supposed to be equally distributed into 2 dissipative components of area $10 \times 10 \mathrm{~mm}^{2}$ each and one dissipative component of area $20 \times 20 \mathrm{~mm}^{2}$. The maximum temperature difference reaches $20 \mathrm{~K}$. Just as an illustration, one can note that an aluminum plate $\left(\lambda=165 \mathrm{Wm}^{-1} \mathrm{~K}^{-1}\right)$ of thickness $13 \mathrm{~mm}$ (i.e. more than 4 times thicker than a FPHP of $3 \mathrm{~mm}$ ) would present the same thermal performance (Fig.20). The maximum temperature is reached under the small heat sources, where the heat flux is the highest.

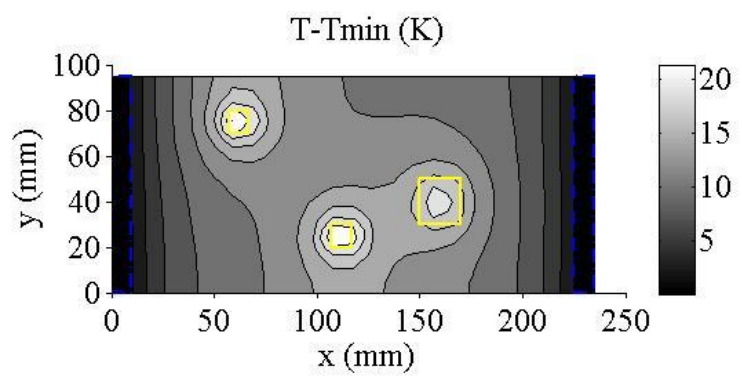

Fig. 19 Temperature field on the FPHP with 3 electronic components

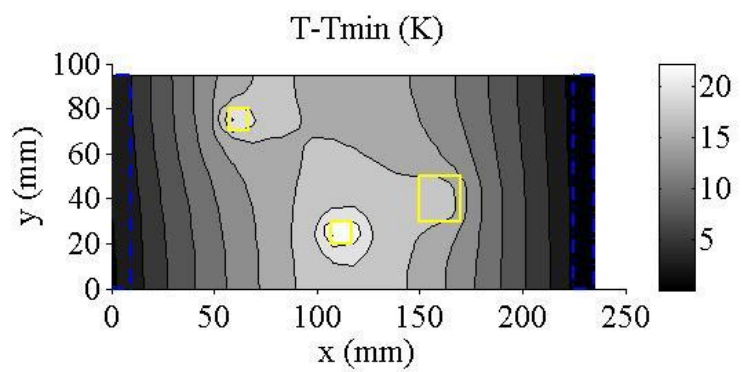

Fig. 20 Temperature field on an aluminum plate of thickness $13 \mathrm{~mm}$
Fig. 21 presents the liquid pressure field inside the screen meshes. The maximum pressure difference inside the capillary structure is lower than $1700 \mathrm{~Pa}$, which is below the capillary limit $(2000 \mathrm{~Pa})$.

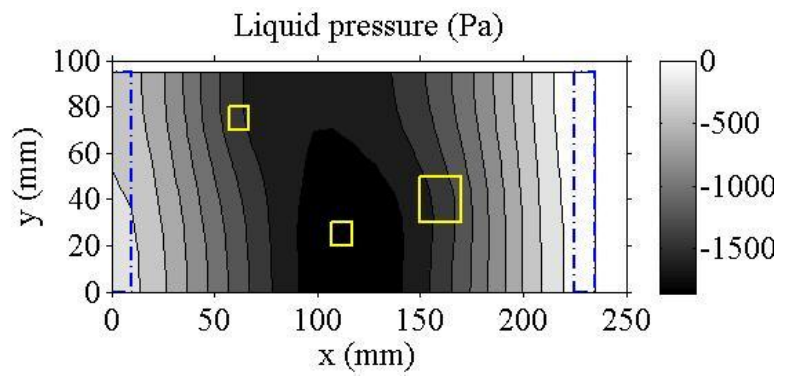

Fig. 21 Liquid pressure in the screen meshes

\section{CONCLUSIONS}

A synthesis of observations made inside the capillary structure of six different flat plate heat pipes (FPHPs) has been presented. Measurements of the liquid-vapor interface have been provided using confocal microscopy. This non intrusive technique enables the measurements of liquid film of very small thickness (down to $2 \mu \mathrm{m}$ ). These results are very useful to understand the physical mechanisms inside FPHPs that are generally characterized only with temperature measurements, the system being opaque. The results were used to validate FPHP models. The effect of the interfacial shear stress was found to be negligible in all the experimental conditions. On the contrary, we showed that the vapor space thickness has a major influence on the liquid distribution inside the system and thus the thermal performances of FPHPs. It was also shown that nucleate boiling does not constitute an operating limit in grooved heat pipe as it is generally stated in the literature. On the contrary, when meshes are plated against a grooved capillary structure, a strong boiling limit is observed for rather small heat fluxes.

The comparison with an analytical model shows that the equivalent thermal conductivity of the meshes is close to the thermal conductivity of the methanol. This result is not as high as one could expect in comparison with theoretical results. On the contrary, the permeability estimated experimentally is higher than that calculated theoretically. This result confirms that new laws have to be developed to better describe both the permeability and the equivalent conductivity of any kind of capillary structures. Hydrodynamic and thermal simulations show the ability of the flat plate heat pipe to cool several electronic components located on a printed circuit board. The maximum heat transfer rate in horizontal location is around $80 \mathrm{~W}$ for a $0.2 \mathrm{~m}$ long card.

\section{NOMENCLATURE}

$a, b, c \quad$ dimensions of the FPHP (m)

$A_{m n}, B_{m n} \quad$ coefficients for the Fourier series

$C_{1}, C_{2} \quad$ constants

$d \quad$ distance between two fibers (m)

$H \quad$ height (m)

$K \quad$ permeability $\left(\mathrm{m}^{2}\right)$

$N \quad$ number of fibers

$P \quad$ pressure $(\mathrm{Pa})$

$Q \quad$ heat transfer rate $(\mathrm{W})$

$r_{\text {eff }} \quad$ effective radius $(\mathrm{m})$

$T$ temperature (K)

$w \quad$ distance between two fibers (m)

$x, y, z \quad$ coordinates $(\mathrm{m})$ 


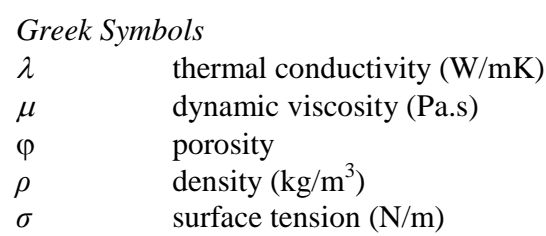

$\begin{array}{ll}\text { Subscripts } & \\ \text { cap } & \text { capillary } \\ e q & \text { equivalent } \\ g & \text { gravity } \\ l & \text { liquid } \\ \max & \text { maximum } \\ p & \text { porous } \\ v & \text { vapor } \\ s & \text { solid } \\ \text { sat } & \text { saturation }\end{array}$

\section{REFERENCES}

Faghri, A., 1995, Heat Pipe Science and Technology, Taylor and Francis, Washington D.C.

Khrustalev, D., Faghri, A., 1995, "Thermal characteristics of conventional and flat miniature axially grooved heat pipes", Journal of Heat transfer, 117, 1048-1054.

http://dx.doi.org/doi:10.1115/1.2836280

Kim, S.J., Seo, J.K., Do, K.H., 2003, “Analytical and experimental investigation on the operational characteristics and the thermal optimization of a miniature heat pipe with a grooved wick structure", International Journal of Heat and Mass Transfer, 46, 2051-2063. http://dx.doi.org/doi:10.1016/S0017-9310(02)00504-5

Lallemand, M., Lefèvre, F., 2004, "Micro/mini heat pipes for the cooling of electronic devices", Proceedings of $13^{\text {th }}$ International Heat Pipe Conference, Shanghai, China.

Lefèvre, F., Lallemand, M., 2006, "Coupled thermal and hydrodynamic models of flat micro heat pipes for the cooling of multiple electronic components", International Journal of Heat and Mass Transfer, 49, 1375-1383.

http://dx.doi.org/10.1016/j.ijheatmasstransfer.2005.10.001

Lefèvre, F., Rullière, R., Pandraud, G., Lallemand, M., 2008, "Prediction of the temperature field in flat plate heat pipes with microgrooves - Experimental validation", International Journal of Heat and Mass Transfer, 51, 4083-4094.

http://dx.doi.org/doi:10.1016/j.ijheatmasstransfer.2007.12.007

Lefèvre, F., Rullière, R., Lips, S., Bonjour, J., 2010, "Confocal microscopy applied to capillary film measurements in a radial flat plate heat pipe made of silicon", Journal of Heat Transfer, 132(3), 031502 http://dx.doi.org/doi:10.1115/1.4000057
Lefèvre, F., Conrardy, J.B., Raynaud, M., Bonjour, J., 2011, “A flat plate heat pipe with screen meshes for the cooling of electronic components", Proceedings of $10^{\text {th }}$ International Heat Pipe Symposium, Taipei, Taiwan.

Lefèvre, F., Conrardy, J.B., Raynaud, M., Bonjour, J., 2012, "Experimental investigations of flat plate heat pipes with screen meshes or grooves covered with screen meshes as capillary structure", Applied Thermal Engineering, 37, 95-102. http://dx.doi.org/10.1016/j.applthermaleng.2011.11.022

Lips, S., Lefèvre F., Bonjour J., 2009, "Nucleate boiling in a flat grooved heat pipe", International. Journal of Thermal Science, 48, $1272-1278$ http://dx.doi.org/doi:10.1016/j.ijthermalsci.2008.11.011

Lips, S., Bonjour, J., Lefèvre, F., 2010a, "Investigation of evaporation and condensation processes specific to grooved flat heat pipes", Frontiers in Heat Pipes, 1, 023001. http://dx.doi.org/10.5098/fhp.v1.2.3001

Lips, S., Lefèvre, F., Bonjour, J., 2010b, "Combined effects of the filling ratio and the vapor space thickness on the performance of a flat plate heat pipe", International Journal of Heat and Mass Transfer, 53, 1113-1121.

http://dx.doi.org/doi:10.1016/j.ijheatmasstransfer.2009.10.022

Lips, S., Lefèvre, F., Bonjour, J., 2010c, “Thermo-hydraulic study of a flat plate heat pipe by means of confocal microscopy: application to a 2D capillary structure", Journal of Heat Transfer, 132, 112901. http://dx.doi.org/10.1115/1.4001930

Lips, S., Lefèvre, F., Bonjour, J., 2011, "Physical mechanisms involved in grooved flat heat pipes: experimental and numerical analyses", International Journal of Thermal Sciences, 50, 1243-1252. http://dx.doi.org/10.1016/j.ijthermalsci.2011.02.008

Revellin, R., Rullière, R., Lefèvre, F., Bonjour, J., 2009, “Experimental validation of an analytical model for predicting the thermal and hydrodynamic capabilities of flat micro heat pipes", Applied Thermal Engineering, 29, 1114-1122. http://dx.doi.org/10.1016/j.applthermaleng.2008.06.002

Rullière, R., Lefèvre, F., Lallemand, M., 2007, "Prediction of the maximum heat transfer capability of two-phase heat spreaders Experimental validation", International Journal of Heat and Mass Transfer, 50, 1255-1262. http://dx.doi.org/doi:10.1016/j.ijheatmasstransfer.2006.09.015

Tibiriça, C. B., Do Nascimento, F. J., Ribatski, G., 2010, "Film thickness measurement techniques applied to micro-scale two-phase flow systems", Experimental Thermal and Fluid Science, 34, 463-473 http://dx.doi.org/10.1016/j.expthermflusci.2009.03.009

Vasiliev, L.L., 2008, "Micro and miniature heat pipes - Electronic component coolers", Applied Thermal Engineering, 28, 266-273. http://dx.doi.org/10.1016/j.applthermaleng.2006.02.023 